\title{
Reading the city through its neighbourhoods: Deep text embeddings of Yelp reviews as a basis for determining similarity and change
}

\author{
Alexander W Olson ${ }^{\mathrm{a}, \mathrm{d}, \mathrm{c}}$, Fernando Calderón-Figueroa ${ }^{\mathrm{b}, \mathrm{d}}$, Olimpia Bidian ${ }^{\mathrm{b}, \mathrm{d}}$, and \\ Daniel Silver ${ }^{\mathrm{b}, \mathrm{d}}$, Scott Sanner ${ }^{\mathrm{a}, \mathrm{d}}$ \\ ${ }^{a}$ Department of Mechanical and Industrial Engineering \\ ${ }^{b}$ Department of Sociology \\ ${ }^{c}$ Corresponding Author-aolson@mie.utoronto.ca \\ 40 St George St, Toronto, ON M5S 2E4 \\ ${ }^{d}$ University of Toronto, Canada
}

\begin{abstract}
This paper develops novel methods for using Yelp reviews as a window into the collective representations of a city and its neighbourhoods. Basing analysis on social media data such as Yelp is a challenging task because review data is highly sparse and direct analysis may fail to uncover hidden trends. To this end, we propose a deep autoencoder approach for embedding the language of neighbourhood-based business reviews into a reduced dimensional space that facilitates similarity comparison of neighbourhoods and their change over time. Our model improves performance in distinguishing real and fake neighbourhood descriptions derived from real reviews, increasing performance in the task from an average accuracy of 0.46 to 0.77 . This improvement in performance indicates that this novel application of embedded language analysis permits us to uncover comparative trends in neighbourhood change through the lens of their venues' reviews, providing a computational methodology for reading a city through its neighbourhoods. The resulting toolkit makes it possible to examine a city's current sociological trends in terms of its neighbourhoods' collective identities.
\end{abstract}

Keywords: Computational Social Science, Urban Informatics, Neighbourhood Analysis, Machine Learning, Text Embedding

\section{Introduction}

Until recently, people learned about cities mostly through personal experience, conversations, travel guides, and other printed material in newspapers and Preprint submitted to Cities

December 3, 2020 
4 books. The advent of the Internet has tremendously expanded the number of others from whom we can learn about what a city has to offer, and how easily we can access this information. Indeed, we now routinely receive recommendations from millions of people through consumer review websites such as Yelp.com. These websites are designed to provide consumers with information about the quality of various amenities they may wish to try, while also offering them a chance to participate in an ongoing conversation about those amenities.

The millions of reviews accumulated in the process also provide researchers with rich data for uncovering how people interpret and evaluate the context around them - provided we can devise the tools necessary to translate this information into a form by which we too can 'read' [1, 2, 3] the city. In this paper, we demonstrate via a case study of Toronto, Canada, that it is possible to characterize neighbourhoods using Yelp review data and examine their relation to each other. We propose novel computational methods for reading the city through the massive corpus generated and compiled by users of these websites and show that the resulting representation captures the evolving discourse by which users represent to one another what various parts of a city mean to them. In so doing, we combine concepts traditionally associated with qualitative and phenomenological approaches to urban life [4, 5, 6]-legibility, interpretation, discourse, meaning-with novel computational methods that allow us to investigate them systematically, comparatively, and in a way that is reproducible by others. By no means do we believe that this "thin description" can or should replace the traditional tools of "thick description." However, we do suggest that the two can work in tandem and that a significant contribution of this paper is to illustrate that the qualities and meanings of urban life can be examined quantitatively.

To meet this goal, we introduce an embedding-based computational approach to analyzing the discursive transformation of a city over time, chiefly at the neighbourhood level. We work with a dataset comprising all Yelp reviews for the city of Toronto over ten years. To draw a quantitative analysis of the change in neighbourhoods from these reviews, we introduce an autoencoder-based methodology, which learns a compact embedded representation of review data from relatively sparse word usage at the surface review level. We then go on to demonstrate that this compact representation, when applied to aggregated reviews at the neighbourhood level, contains critical information about each neighbourhood, which can be used to investigate how neighbourhoods change over time. By separating reviews by the year in which they were written, we employ our embedding space to examine how neighbourhoods change relative to each other. We identify a set of very stable neighbourhoods and use the Most Stable Neigh- 
bourhood (MSN) to find categories of change.

These methodological innovations are the central contribution of the paper. However, they point toward a significant substantive contribution as well: the capacity to identify stable and dynamic neighbourhood collective identities using the actual textual artifacts of neighbourhood users that could be scaled up more broadly. If economists have used similar data as a way to monitor economic trends in a way that goes beyond what standard official indicators permit (e.g. [7, 8, 9]), our methods offer techniques for using Yelp data to monitor sociological trends in a large-scale way, even where sources such as survey data are unavailable.

To our knowledge, no previous study on neighbourhood change has exploited the richness of data offered by Yelp reviews - i.e., both the attributes of geolocated venues and the underlying structure of review texts. Capitalizing on these features, we aim to answer two questions:

1. Do Yelp review data allow us to identify neighbourhood boundaries using text analysis and machine learning techniques?

2. By aggregating Yelp reviews by neighbourhood and year, is it possible to identify neighbourhood change at the discursive level? If so, is it possible to identify which neighbourhoods become more similar or more different to one another over time?

Section 2 reviews prior attempts to "read" the city, existing research using Yelp and other social media data, and relevant work on computational methods for large-scale latent text analysis. Sections 3 and 4 present our analytical approach, methods, and results. We conclude with a discussion of the broader significance of this research, its limits, and future directions.

\section{Literature Review}

\section{Reading the City's Neighbourhoods}

The neighbourhood is one of the most distinctive features of a city. Neighbourhoods are not only crucial for people's cognitive maps and their sense of identity, but they are increasingly central targets for planning and social policy [10]. Therefore, a proper account of the boundaries of a city's neighbourhoods is not just a matter of scholarly interest but has significant social and political consequences. Neighbourhood boundaries are generally difficult to establish. Spatial features alone have proven to be ineffective in identifying boundaries that local people can distinguish accurately [11, 12]. While official neighbourhood boundaries drawn by municipal authorities do not always reflect existing 
communities [13], they are often used for policy, real estate, and marketing purposes [14]. Official boundaries accordingly offer a useful baseline to test how accurately 'big data' can identify neighbourhoods in a city.

Building on Suttles [6], a stream of urban research approaches the neighbourhood as a creative imposition that sits in the intersection between the physical environment, both internal and external to its boundaries, and people's interactions on it. People's activities in the city are closely connected to their 'cognitive maps' - their internal representations of the space of the city. People develop cognitive maps beyond their neighbourhoods of residence as they move around the city either on their way to work or for other purposes [15]. Thus, the map of a single neighbourhood is the result of an ongoing conversation among residents and external agents, such as occasional visitors, local authorities, and developers. As a result, the "common knowledge" about what a neighbourhood's identity consists of is a collective representation that transcends the interpretation of any single individual or group. This is a pressing issue in contemporary neighbourhood scholarship. In her recent book, Talen [16] identifies several crucial features of neighbourhood efficacy. One of these is a coherent shared identity that residents and others can identify and rally around, as well as various meeting places and venues that affirm and support this meaning. However, a systematic reconstruction of cognitive maps, particularly the neighbourhood boundaries within them, poses several methodological challenges. Some survey and interview-based studies rely on mapping exercises administered to residents. The data obtained is later processed to identify either the "consensus map," the average, or the largest resident-defined boundaries [17]. These techniques are expensive and difficult to validate [18, 19].

Among the earliest attempts to draw cognitive maps from interview-based mapping evidence, Lynch's [5] work is one of the most influential to this day (cf. [12, 20]). Lynch's point of departure is that cities are legible—human beings are capable of recognizing different parts of cities and organizing them as coherent patterns. One type of pattern Lynch discusses is the district: each two-dimensional area of the city whose boundaries are recognizable through a "common, identifying character." Although districts are not necessarily neighbourhoods, they allow us to think about neighbourhoods beyond people and residential infrastructure, and to pay attention to the configuration of businesses and public places that can give a sense of coherence to a specific portion of the city. Recently, Filomena and colleagues [12] tried to reproduce Lynch's cognitive map of Boston using GIScience techniques. To identify district boundaries, they relied on modularity optimization to analyze the road layout of contempo- 
rary Boston. Their findings were modest in relation to district identification due to the lack of data about people's activities in the space.

Studies that rely mostly on physical features to characterize neighbourhood boundaries do not aptly capture the meanings attached to such features. Lynch's [5] metaphorical link between the city and language has been extended by more recent studies that aim to read the city as text, which typically implies some form of semiotic practice-i.e., the practice involved in meaning-making. For instance, for Barthes [4] analyzing the discourse around the topics of interest in a city is an essential complement to the survey and interview data traditionally used. Other authors [21, 22] look at the language of the city in a manner similar to reading works of literature. Still others employ the paratextual meanings of advertising [23], interpretations of art and architecture [24], or landscapes [25]. Nonetheless, the nature of the data used, combined with the interpretation-intensive methods proposed, limits the scope of these studies substantially.

\section{Big Data and Social Media as Tools for Reading the City}

Contemporary big data has the potential to identify linguistic patterns in the city in a more systematic fashion, even if this possibility has not yet been fully realized. Twitter data for example has been used in many geographical analyses [26, 27], for instance, to explore the degree of geographic concentration of virtual social networks [28], to predict intrinsic population characteristics (including the geographical location of the authors) from food-related tweets [29], and to map linguistic communities in a cosmopolitan city like Milan [30] and "city communities" through machine learning techniques in Bogotá [31]. In a recent study, researchers used geo-located tweets to measure the social integration of U.S. cities based on the everyday travels of people across neighbourhoods [15]. It is worth noting that Twitter data, even when georeferenced, is limited in its geographic scope since there is no guarantee that a tweet posted from a given location is about that location. Nonetheless, as with earlier studies [15], our method is applicable to Twitter just as it is applicable to Yelp. The method works with any large-scale text corpus, we have simply selected Yelp as the resource to apply it on in this paper.

Other location-based services have provided an avalanche of data for urban and regional scholarship, but these too are not always ideal for uncovering patterns of urban discourse. Foursquare data for example has been used to locate groups of users in the city by analyzing the places they visit [32], to distinguish cultural boundaries by looking at food and drink habits [33, 34], to identify the sources of urban "buzz" [35], to identify neighbourhood boundaries though patterns of collective activities and social proximity [11, 36, 33], and in urban plan- 
ning to allocate retail stores in the city better [37]. In relation to neighbourhood attributes and change, Foursquare data has been compared to traditional spatial data (e.g., property maps) to study the relationship between the presence of specific physical venues, such as tanning salons or flea markets, and the neighbourhood socioeconomic deprivation [38, 28]. Other studies have shown that Foursquare-and similar location-based services-accurately predict changes in local socioeconomic attributes, which is especially useful for regions with unreliable or delayed official data sources [8, 9]. Urban patterns have been extracted from other types of georeferenced data [39]. A notable example is the Streetscore method, which employs deep learning to extract information about the perceived quality of an area using Google StreetView data [40].

Nevertheless, Foursquare and similar services are limited for the purpose of reading the city due to the lack of user-generated text. Additionally, Foursquare's categories are not fully structured - the categorization of venues is not always reliable. Overall, while neighbourhood change is a recurrent topic of analysis that has received new energy as novel data and techniques allow long-term trajectories to be analyzed systemically, the discursive meanings associated with neighbourhood change are difficult to capture beyond case studies. There is abundant research on gentrification and displacement focused on changes in the demographic composition of neighbourhoods, and changes in their built environment - e.g, the spread of cafés. Other studies that explore the experiences of gentrification by residents are usually limited in geographic scope.

Yelp reviews, by contrast, provide a large dataset on how people talk about neighbourhoods. Yelp offers multiple distinctive data attributes that have been exploited in previous research, though this neighbourhood dimension has not been featured to our knowledge. For example, studies have used natural language analysis tools to test the relationship between language choices and the rating given to a venue on Yelp. For instance, Jurafsky and colleagues extract the narrative framings more commonly associated with positive and negative reviews of restaurants [41, 42]. Other studies show how the use of expressions of authenticity in reviews is usually associated with higher ratings [43]. The rich categorization of Yelp venues, particularly restaurants, allows us to categorize its users according to consumption patterns and explore their likelihood to value "pure" versus "transgressive" cultural objects [44].

Yelp data has been used for neighbourhood change and gentrification research, albeit not to its full potential. Yelp's record of active venues has been used to monitor changes in the local economy of neighbourhoods, complementing the lagged official data such as County Business Patterns in the U.S. [7]. 
Glaeser and colleagues [45] also used Yelp reviews of coffee shops as proxies of change in the local economy of neighbourhoods associated with gentrification. The findings suggest that Yelp data can provide real-time and up-to-date insights into the changes in the neighbourhood economy and gentrification before the official statistics are released. Studies on neighbourhood change that do engage with review texts have limitations due to the nature of the methods they use. Zukin, Lindeman and Hurson [46] identify racial bias in comparing the Yelp reviews of restaurants in two gentrifying neighbourhoods-one predominantly Black and another predominantly White. Their study, however, is limited to 7046 reviews, since they use manual coding to capture terms that show the implicit racial bias.

In sum, our brief survey indicates that Yelp data has the potential to make the abstract notion of a neighbourhood's collective representation by myriad individuals empirically tractable. Yelp reviews offer a set of advantages for the identification of geographic and linguistic patterns given that all information is explicitly about a specific location in the city, known by both the user and the researcher. Just as important, this dataset captures changes over time. We can therefore identify when certain discourses emerge and how they spread, using the tools of modern computational text analysis. Ultimately, neighbourhood changes matter the most because they affect peoples' experiences in the city.We aim to show how new data and methods can incorporate these experiences into quantitative studies of neighbourhood change.

\section{Computational Methods for Large-scale Latent Text Analysis}

To uncover the discursive structures associated with neighbourhoods in the Yelp data, we apply and extend computational techniques using large-scale social media data to understand city dynamics [11]. This literature has addressed the issue of recognizing patterns of discursive stability and change in cities through longitudinal analysis of social media text. While a full review is beyond the scope of this paper, we focus specifically on latent analysis methods that aim to reduce large volumes of textual data to low dimensional representations that preserve meaning and facilitate measurement of semantic similarity.

A first class of latent text analysis methods relate to clustering and topic modeling methods that seek to assign explicitly interpretable latent labels to "documents". In the context of large-scale social media data in an urban setting, a document may consist of an aggregation of all text content relating to a neighbourhood, an individual, a business, a time period, or any other observable factors (or combination thereof). Clustering text documents using $K$-means has a long history [47] and assumes that every document belongs to one of $K$ latent 
classes (or types); by analyzing the word frequencies of text with the same latent class assignment, it is often possible to understand the meaning of clusters through their most frequent words. In a related vein, others use unsupervised topic modelling methods such as Latent Dirichlet Allocation [48], increasingly common in social sciences [49, 50], to examine semantic trends in urban spaces (e.g. [51]). Unlike clustering, topic modeling allows a single document to have multiple labels to varying degrees. One goal of topic modeling in this context is to ascribe each neighbourhood to one or more topic labels that can be understood according to the vocabulary distribution learned for each topic.

A second type of study focuses on machine learning and deep learning methods to encode documents or words into low dimensional latent spaces that lack explicit interpretation, but which facilitate semantic similarity comparison of embedded content. Two of the standard models for word embeddings, GloVe [52] and word2vec [53], transform words from English to a vector-based encoding. This encoding captures the meaning of each word by positioning it in embedding space, such that the distance between any two words corresponds to their similarity. These approaches, then, create an embedded space for words to exist within, such that the exact position of the word captures essential information about its meaning. These methods can also be extended to documents as in doc2vec [54].

Our paper builds on this second type of embedding approach through a novel application of an autoencoder model on geolocated textual data. While the architecture of word and document embedding models diverge somewhat from a standard autoencoder model, it is well established that the latter is strongly suited to extracting low-dimensional embedded representations of text that retain the underlying meaning and facilitate semantic similarity analysis (e.g. [55, 56, 57]). This allows us to identify geographically-anchored semantic changes over time through the similarity computations enabled by the use of embeddings.

\section{Data and Methods}

\section{Data}

Our study uses data from Yelp.com. Yelp is a popular consumer review website. For instance, it offers approximately 163 million reviews, in 18 different languages, and the average number of unique website visitors is around 150 million [58]. Over 92\% of Yelp users are from the United States, with Japan, India, and Canada as its next largest, but at a modest $1 \%$ per country. In addition to reviews, Yelp provides information and reminders about local events, lists of local businesses and their offers, can make restaurant reservations, and allows those who have an account to connect with (i.e. follow) other 'Yelpers' 
As with many social media data sources [59], Yelp represents an unbalanced sample of the total population of the cities it covers ${ }^{1}$ Yelp nevertheless tracks differences between real neighbourhoods, as we show below. It should also be noted that Yelp data cannot be construed as representative of a particular geographically defined population, which may pose concerns in terms of external validity and generalizability. For this study, the location of reviewers is irrelevant, as the main goal is to analyze changes in the reviewers' language, to understand better how city neighbourhoods are represented and change over time.

Yelp is attractive as a data source for many reasons. In particular, it contains rich information about (a) points of interest (POI) and (b) qualitative assessments of these POI (reviews). POIs are mainly businesses, but also may include public spaces such as parks or hiking trails. For each POI listed, there is a large amount of data available describing its location, opening hours, and other minutiae. Each POI is classified within a hierarchical list of approximately 1200 categories and by ambience (e.g. 'family-friendly' or 'romantic'). These characteristics are valuable in learning about the type of features available in different areas, yet the reviews add more: qualitative and evaluative assessments. These combine a star rating (out of five) with a review written by the user. The reviews are date stamped and correspond to a discrete location, enabling geospatial and temporal analysis.

We have collected Yelp data for the city of Toronto, which we use as a case study for developing methods that should be applicable elsewhere. The dataset includes approximately 24,000 businesses and 500,000 reviews. This number was validated against results from [7], which found that across the US, Yelp had $18.7 \%$ of the listings that the official business patterns dataset did. Our Toronto dataset comprises a similar proportion of all businesses officially registered in Toronto, which includes many non-consumer facing businesses that would not be expected to have Yelp pages. In addition to the count of establishments, our dataset also includes information about each POI's category (e.g. 'sushi restaurant' or 'rock climbing') and all of its reviews. Each review is also indexed to the

\footnotetext{
${ }^{1}$ In terms of demographics, Yelp reviewers are relatively equally represented within the 18-34 and 35-54 age groups (i.e., standing at approximately 35\%), with the 55+ age group representing about $30 \%$. Furthermore, over $60 \%$ are college-educated, and an additional $23 \%$ have a graduate degree. Concerning income, almost half make over $\$ 100,000$ annually, and another $25 \%$ are in the $\$ 60,000-99,000$ range. These numbers suggest that while Yelp reviews capture a broad swathe of the population, as with other Internet data, they skew toward the younger and more educated (e.g. [60, 61] ). Information about the gender distribution, as well as other attributes among Yelp users, has not been made available by the company. However, independent studies indicate that Yelp's user base is composed slightly more of women than of men [62, 63].
} 
reviewer who wrote it. Finally, we have a Business ID, a User ID, and a location (lat/lon) associated with each review.

\section{Analytical Approach}

In order to suitably represent neighbourhoods, we adopt a standard termfrequency bag-of-words (BoW) representation (as seen in e.g. [64, 65, 66]). This method indicates only the presence of words and discards their order. We could compute similarity directly on these vectors, but as they are sparse, this would not capture the different ways linguistic expressions may refer to the same things. To address this, Deerwester [67] established Latent Semantic Analysis (LSA) as a means to apply Principal Component Analysis to linearly project sparse vectorbased data to a lower-dimensional dense representation where semantically similar expressions are located nearby in vector space. In the modern era of deep learning, we can now learn non-linear embeddings from large datasets that can better exploit the non-linear relationship between word co-occurrence to produce improved low-dimensional representations for similarity comparison. In order to compute the similarity metric over neighbourhoods, we employ Cosine Similarity, which is the standard metric for any vector-based representation [47].

To answer our first research question - do Yelp review data allow us to identify neighbourhood boundaries using text analysis and machine learning techniques? - we introduce the concept of the autoencoder, and describe how our data is formatted to train the model. We then explain the underlying concept of latent embedding space. Using these concepts, we show that Yelp review texts accurately identify the language patterns characteristic of the City of Toronto's 140 official neighbourhoods (Figure 1), despite having not been trained on data directly about them. In order to demonstrate the validity of this result, we conduct a "fake neighbourhood experiment," in which our model is tasked with distinguishing "real" and "fake" neighbourhoods. We find it does so with a high degree of accuracy, especially in contrast to alternatives.

To answer our second research question - is it possible to identify neighbourhood change at the discursive level? If so is it possible to identify which neighbourhoods become more similar or more different to one another over time? - we aggregate the Yelp data into vectors representing individual reviews $(R)$, businesses $(B)$, users $(U)$, and neighbourhoods $(N)$. We then break up these vectors by year to create a platform for temporal analysis. We embed these units in the same space as the atemporal units so that we can track the motion of a single object - the linguistic patterns of a single neighbourhood - over time. By embedding the temporal neighbourhood objects in a single space, we can construct a concept of stable vs unstable neighbourhoods. Stable neighbourhoods 


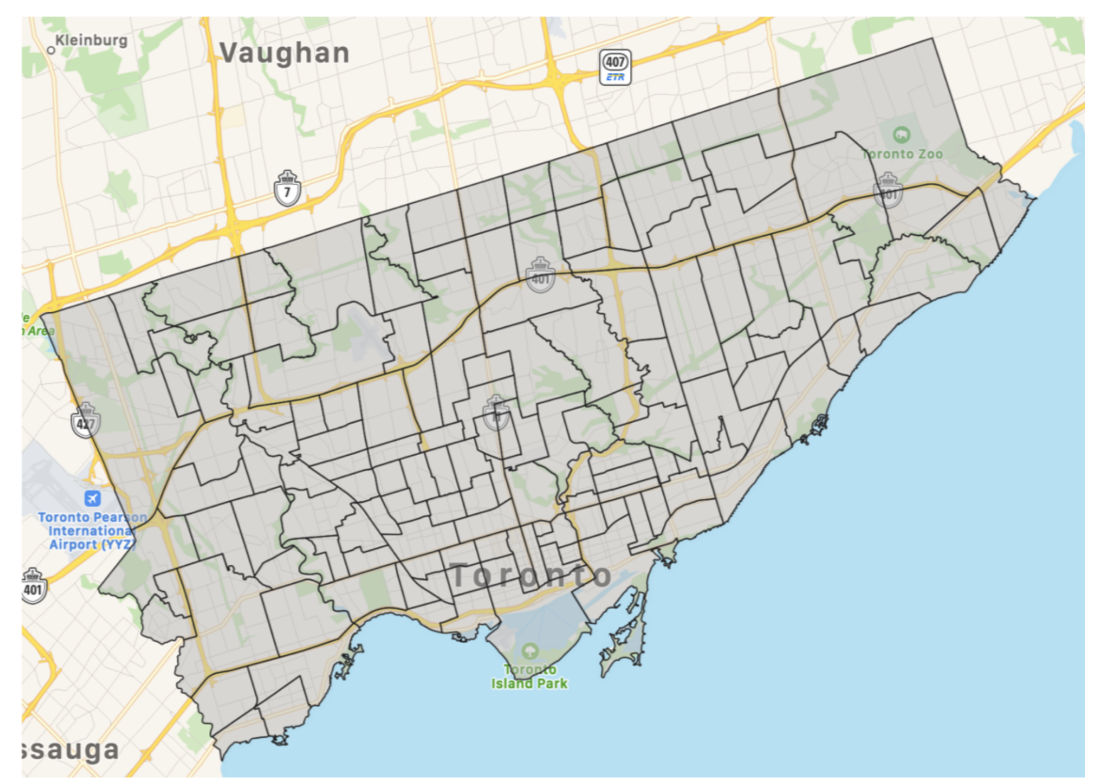

Figure 1: The 140 officially-defined neighbourhoods of Toronto.

do not move much in the embedding space year-on-year, while unstable neighbourhoods move rapidly. We treat the stable neighbourhoods as anchors and examine how other neighbourhoods change relative to them. Finally, we employ K-Means clustering to identify different groups of motion through the resulting proximity space, such as those neighbourhoods which are rapidly changing, or well-established downtown neighbourhoods which, despite being home to some of the city's most dynamic cultural scenes, change little in terms of their collective meaning. This approach allows us to read the city in terms of how neighbourhoods function as templates or models that others become more or less similar to over time and to classify urban text by its patterns of evolution.

Figure 2 summarizes the encoding process. The concrete steps taken, from cleaning and vectorizing the raw data to performing neighbourhood recognition experiments, are detailed in the following subsections. 


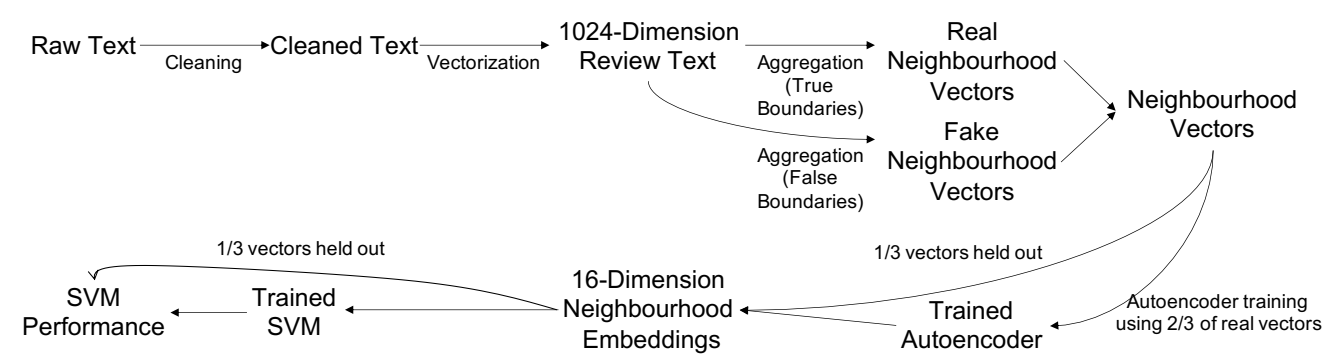

Figure 2: Methodology pipeline from raw review data to the result of the first experiment. For the baseline comparison, the autoencoder step is omitted and the full 1024-dimension vectors are used in the Support Vector Machine (SVM) step.

\section{Cleaning and Vectorization}

The standardization of review text is initially noisy and somewhat unreliable. We employ a natural language processing tool, spaCy [68], to extract standard word tokens from reviews. Using spaCy, we can transform the raw text into a set of standard words. We then remove accents, as well as stop words, which are non-informative words such as "the," "of" or "and." We also omit any words which occur in less than 100 distinct reviews.

We then convert each review into a 1024-long vector of integers. Each value $i$ in this vector corresponds to the $i^{\text {th }}$ most commonly used word, or pairs of words, in the dataset, and its value corresponds to the number of times that word is observed in the review. Our selection of 1024 words is not arbitrary - it has been shown [69] that in any large corpus, a tiny proportion of the words available can account for a considerable proportion of the words used. In our case, the 1024 most frequently used words account for approximately $70 \%$ of all words used in reviews. We consider pairs of words as well, in order to include some common phrases in our dataset. Of the 1024 words used, 54 are word pairs. Word pairs include phrases such as 'ice cream', 'pad thai' or 'years ago'.

From our 1024-dimension vectors representing each review, we then construct vectors representing users $(U)$, individual reviews $(R)$, businesses $(B)$, and neighbourhoods $(N)$. For each of these, we simply add all of the review vectors corresponding to that subject (e.g. a single business). We normalize these vectors to sum to 1 , so that the size of the vector does not correspond to review length. 
I had a good experience at this store. I was going to order online but instead thought I'd ask a few questions before making the purchase. I received good and knowledgeable responses and advice, and elected to make the order through the store rather than online. It was an easy process. (I ultimately picked up the mattress instead of opting for free delivery since delivery was booking out farther than we would have liked.)The actual mattress we chose - The Bloom Cloud - is super comfy and we are pleased with the quality. The mattress comes compressed in the box and you can easily set it up on your own. It is also feels really light once open, \xa0which makes moving it around and making the bed easy. I would shop here again and would recommend this store.

Figure 3: The raw review text. good experience store going order online instead thought ask questions making purchase received good knowledgeable advice make order store online easy process ultimately picked instead free delivery delivery booking actual chose super comfy pleased quality comes box easily set feels really light open makes moving making bed easy shop recommend store

Figure 4: The same review text, after the entire cleaning and vectorization process.

\section{Dimensionality Reduction}

After obtaining the review vectors for all categories of objects we wish to analyze, the final stage is reducing the dimensionality of the vectors. This step allows us to 'combine' words in the vector which have similar meanings, or almost always co-occur. Examples of such words are entries such as 'making' and 'makes'. We select a dimensionality reduction to $2^{4}$, i.e. 16 dimensions. In order to accomplish this task, we employ a standard fully-connected autoencoder, the structure of which is shown in Figure 5

\section{Distinguishing Real and Fake neighbourhoods}

In order to demonstrate the validity of the resulting 16-dimensional vector representations of neighbourhoods, we devised an experiment that requires meaningful information to be included in the vectors. This experiment requires a 


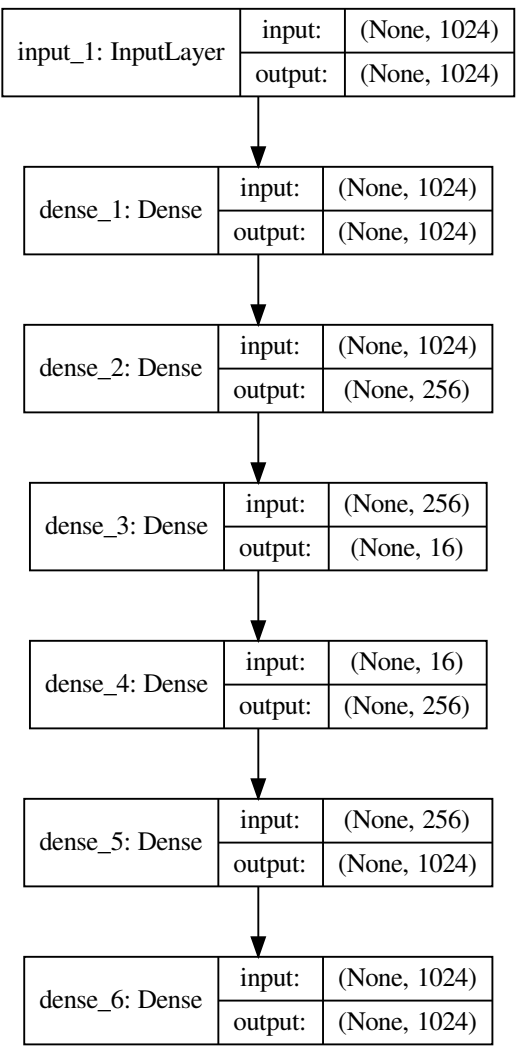

Figure 5: The structure of the Autoencoder model used. The first 3 dense layers, together referred to as the Encoder, transform the full 1024-dimensional vector into a compact, 16dimensional representation. The remaining 3 dense layers, known as the Decoder, then use this 16-dimensional representation to reconstruct the original data as accurately as possible. The 'None' value paired with the dimensionality at each layer is due to the fact that any number of 1024-dimensional vectors can be given to the model at a time - each one is still operated on independently of the others. 
classification model to correctly distinguish between the 140 real Toronto neighbourhoods, and a collection of 'fake' neighbourhoods constructed from real data.

To generate our fake neighbourhoods, we elected not only to draw reviews from the original review sets, but also to select reviews from a contiguous region of the city. In this way, the 'fake' neighbourhoods are nearly identical to real neighbourhoods - they comprise a specific region of Toronto and include all of the reviews within this region. The only distinguishing feature is that the fake neighbourhoods are not officially recognized as neighbourhoods (Figure 5).

We use the following constraints to design our methodology for fake neighbourhood generation 2 A total of 140 fake neighbourhoods are generated, so that randomly guessing the veracity of all neighbourhoods would generate an accuracy of approximately $50 \%$, the lowest effective score on this task. We selected a Support Vector Machine (SVM) model for this task ? $^{3}$

1. Fake neighbourhoods have roughly the same area distribution as real neighbourhoods.

2. Fake neighbourhood boundaries are drawn as polygons, with a number of sides corresponding to that of real neighbourhoods.

3. A fake neighbourhood should encapsulate at least one business since there is no neighbourhood that does not contain any businesses.

Once the fake neighbourhoods have been generated, all neighbourhoods are transformed into the low-dimensional space, shuffled, and randomly assigned to the training and testing sets. $67 \%$ of the vectors are assigned to the training set and the remaining third to the testing set. When comparing the similarity of two neighbourhoods in the embedding space, we must select a suitable comparison metric. For this, we use the Cosine Similarity metric that is the standard for text-based analysis [47], which is defined for two vectors $A$ and $B$ as follows:

$$
\cos (\theta)=\frac{\sum_{i=1}^{N} A_{i} B_{i}}{\sqrt{\sum_{i=1}^{N} A_{i}^{2}} \sqrt{\sum_{i=1}^{N} B_{i}^{2}}}
$$

\footnotetext{
${ }^{2}$ Our full code for this process can be found in the codebase hosted on GitHub.

${ }^{3}$ SVM models identify the decision boundary which best separates the two classes of data in the reduced dimensionality space. A core benefit of selecting this type of model is that it does not require a significant amount of training data. This is important as we only have a total of 280 samples for the real and fake neighbourhoods. Our SVM was trained using the default parameters provided in the Scikit-Learn package [70], i.e. a regularization constant of 1.0 and a kernel coefficient of 1.76 , and achieved high accuracy during training and validation with a mean training accuracy of 0.87 across 100 runs. This indicated that the model was tuned correctly.
} 


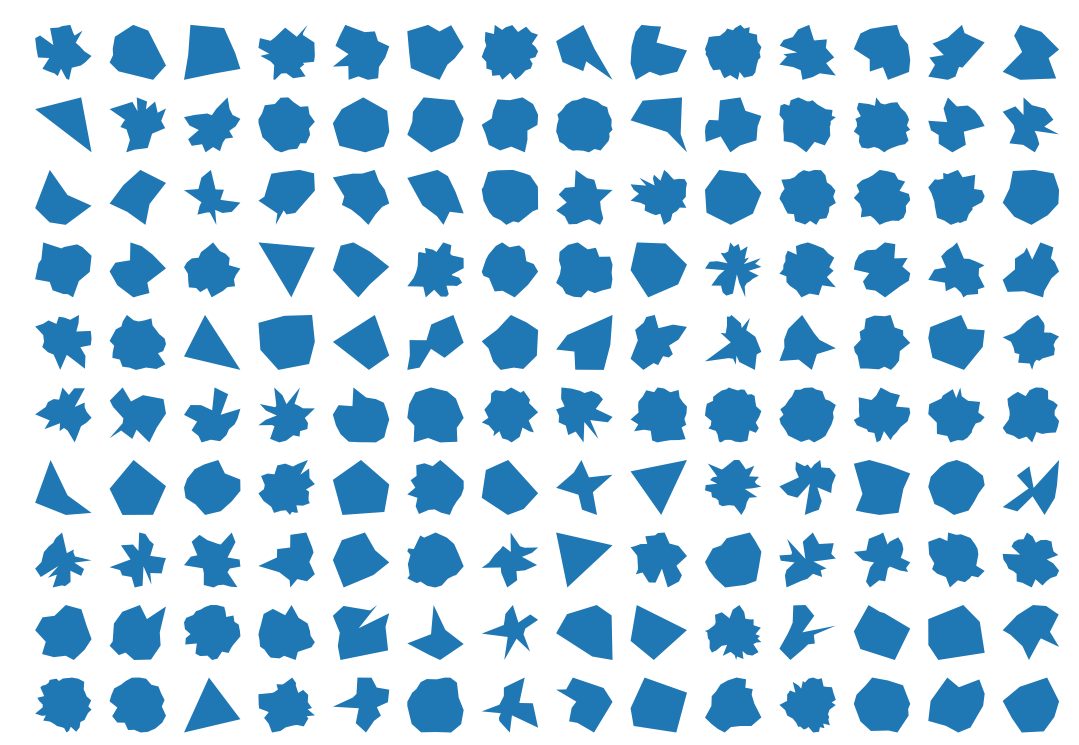

Figure 6: 140 fake neighbourhoods generated using our procedure.

Intuitively, the cosine similarity measures the angle between two vectors, ignoring the length of those vectors. Two vectors pointing in the same direction are identical, and so would have a similarity of 1 , while two vectors pointing in opposite directions would have a similarity of -1 . Orthogonal, or unrelated, vectors have a cosine similarity of 0 .

\section{Results}

\section{Autoencoder Reconstruction Error}

The autoencoder model that was designed for this task was trained on a combination of the vectors representing individual reviews $(R)$, the vectors representing businesses $(B)$, and the vectors representing users $(U)$. As stated earlier, we hold out the neighbourhood vectors $N$ so that, during the fake neighbourhood detection task, the model cannot "remember" that it has seen some of the neighbourhoods before.

As a result of this design, however, it is essential to verify that the model can accurately embed $N$ despite never having been presented with it during training. 
To test this, we pass $N$ through the autoencoder network and record the cosine similarity between the original vector and the reconstructed vector (Table 1).

\begin{tabular}{l|ll}
\hline Data & Cosine Similarity $(\mu)$ & Cosine Similarity $\left(\sigma^{2}\right)$ \\
\hline$R$ & 0.5853 & 0.1331 \\
$B$ & 0.7027 & 0.1525 \\
$U$ & 0.5941 & 0.1294 \\
$N$ & 0.9179 & 0.0538 \\
\hline
\end{tabular}

Table 1: Cosine Similarity between original and reconstructed vectors for $R, B, U, N$. For each result, the worst possible score - indicating total dissimilarity between the original vector and the reconstruction - would be -1 , while the best possible score would be 1 , indicating identity.

SVM Classification Performance

\begin{tabular}{l|ll}
\hline Format & Testing Accuracy $(\mu)$ & Testing Accuracy $(\sigma)$ \\
\hline Full Dimensionality & 0.4630 & 0.0278 \\
Embedding Space & 0.7719 & 0.0341 \\
\hline
\end{tabular}

Table 2: SVM performance in distinguishing real and fake neighbourhoods, over 100 different fake neighbourhood generations. The worst possible score is 0 , however in practice the worst possible performance is around $50 \%$ as this indicates random guesses by the model. The best possible performance is 1 , indicating perfect accuracy.

140 real and 140 fake neighbourhoods were generated, and randomly split into training and testing sets. The SVM model was able to distinguish these real and fake neighbourhoods in the low-dimensional review space with an accuracy of 0.7719 (Table 2).

In the full, 1024-dimensional space, however, accuracy was approximately 0.46 , which indicates random assignment by the classifier. This result is significant for two reasons. First, and most critical, the fact that the real and fake neighbourhoods are indistinguishable in this high-dimensional space demonstrates that the fake neighbourhoods generated are sufficiently 'realistic'. It is vital to be sure that the fake neighbourhoods are not 'too fake' such that any classifier would be able to tell them apart from the real ones. The poor performance of the classifier in this task instead shows that the fake neighbourhoods appear real without proper analysis. Secondly, this result suggests that the structure 

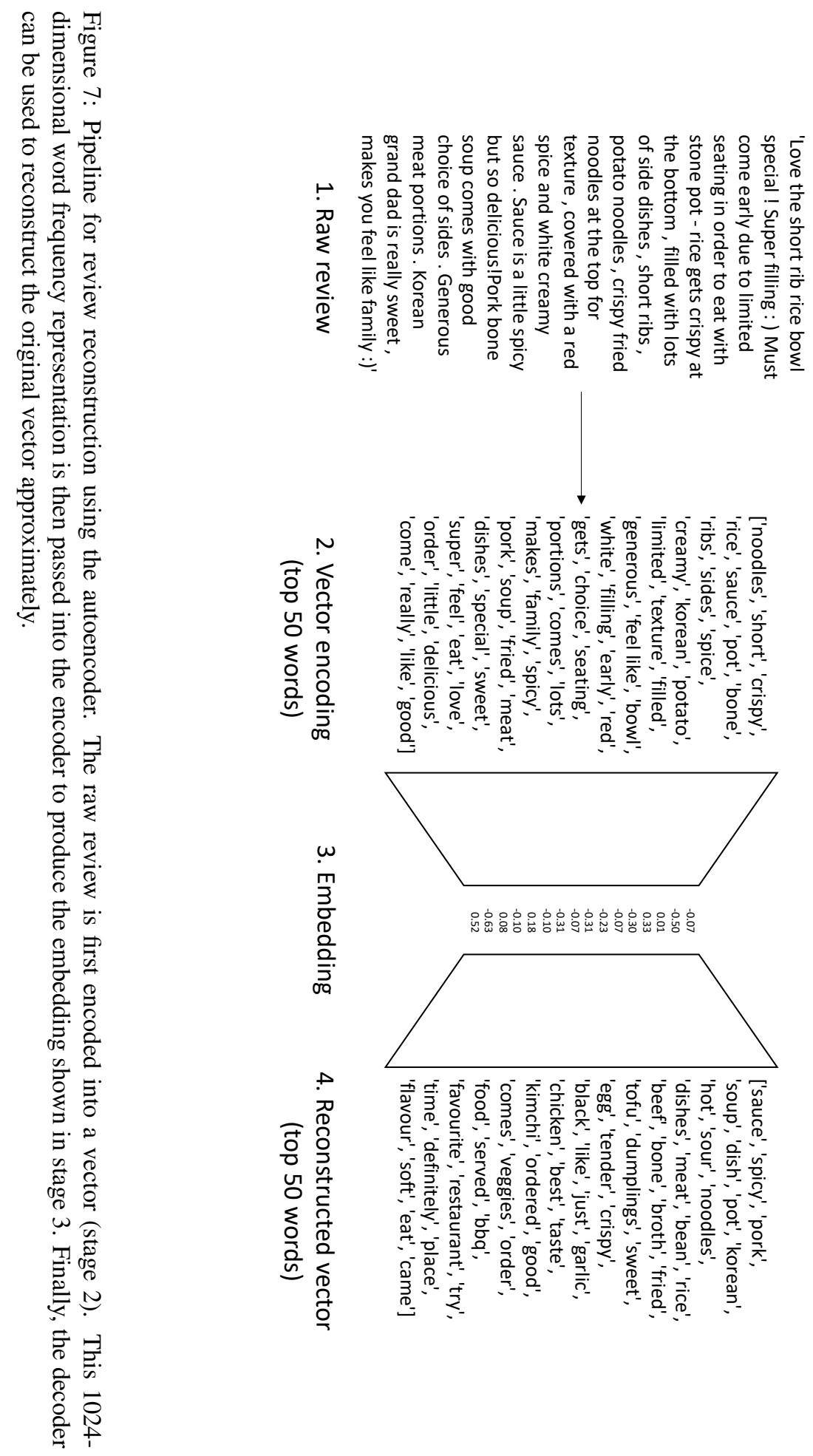
critical to understanding the difference between real and fake neighbourhoods is non-linear and occurs across multiple words in the full space. This inference can be made due to the non-linearity of the Autoencoder model, and the fact that dimensionality reduction is required to distinguish the neighbourhoods successfully.

In sum, the 'fake neighbourhood' experiment indicates that Yelp reviews can be used to identify real neighbourhoods with a high degree of accuracy. In turn, it suggests that Toronto's 140 official neighbourhoods do exhibit relatively distinct styles, and that the official neighbourhood boundaries map to some degree onto underlying differences in how the city is represented. That fake neighbourhoods can be consistently distinguished from real in the latent space, but not in the full word frequency space, suggests that the Autoencoder model is critical to extract the latent information included in the review text.

\section{Interpreting Neighborhood Embeddings}

The embedding space produced by the Autoencoder model is a challenge to analyze. Though we can be sure that the representation learned by the model is a compact representation of the full data, we cannot directly tell what meaning is contained in each of the dimensions in the low-dimensional space. To gain some insight as to the meaning of these dimensions, we provide the second stage of the Autoencoder - the decoder - with a vector in which one dimension is fully maximized, and all other dimensions minimized. By passing such a vector to the decoder, we can learn which words in the full 1024-dimensional space are most strongly associated with each dimension in the encoded representation. For each of the twelve dimensions, we show here the three most strongly associated words (Figure 3). This type of analysis can be referred to as Sensitivity Analysis, as it allows us to examine what aspects of the input data each part of our neural network is sensitive to.

In sum, each dimension of our latent representation captures distinct aspects of what typically defines a business. In depicting the top three words for each dimension here, we demonstrate how our encoder has learned which words essentially mean the same or highly similar things when used to describe a business. In this way, the encoder has deduced that their distinct meanings can be compressed into a single feature. While articulating the full meaning of these dimensions would require going beyond these three-word summaries, in the present context, the simple point is that they do carry distinct meanings that a richer interpretation could seek to unpack. 


\begin{tabular}{l|lll}
\hline Dimension & 1st Word & 2nd Word & 3rd Word \\
\hline 0 & order & delivery & service \\
1 & burger & park & station \\
2 & store & good service & service \\
3 & company & called & phone \\
4 & shop & guys & service review \\
5 & salon & terrible & dress \\
6 & good & place & food \\
7 & tea & cake & cheesecake \\
8 & dress & wedding & store \\
9 & did & went & hair \\
10 & pad thai & nail & nails \\
11 & salon & dress & nails \\
12 & terrible & waited & order \\
13 & bakery & park & cake \\
14 & delicious & atmosphere & cocktails \\
15 & sushi & rice & pho \\
\hline
\end{tabular}

Table 3: The three words with the highest value when a $1 \times 16$ vector containing a single 1 and 15 zeros is passed. Each word triple corresponds to the $K^{\text {th }}$ dimension being given the single 1 . 


\section{Temporal Analysis: Intra-neighbourhood Change}

Having answered our first research question in the affirmative, we now turn to the second. By separating each neighbourhood's reviews by year, and then embedding all reviews into the same embedding space, we can examine the change over time in a neighbourhood's embedded review language. In the following sections, we examine how neighbourhoods change in relation to each other, but first, we study how each neighbourhood changes relative to itself over time.

We define stability as the following for some neighbourhood $\mathrm{N}$ :

$$
\operatorname{stability}(N)=\prod_{i=2}^{|N|} \operatorname{cosin} e_{-} \operatorname{similarity}\left(N_{i-1}, N_{i}\right)
$$

For e.g. four years, can be expanded out to the form:

$$
\frac{A \cdot B \times B \cdot C \times C \cdot D}{\|A\| \times\|B\|^{2} \times\|C\|^{2} \times\|D\|}
$$

We employ this metric, as opposed to an average, in order to represent the "through-line" of semantic similarity. For some neighbourhood with a high interyear similarity, save for two years in which the words used are entirely different, then the average cosine similarity would still be high, losing the insight that at one point the word usage entirely changed. With our metric, however, this single low similarity would affect the entire result, ensuring that no neighbourhood with a high stability value can have any years of dissimilarity within.

For a small set of neighbourhoods, we identified stability below 0.0. These neighbourhoods are noted in Figure 8 as white, and we remove them from further analysis as we consider their motion to be too erratic to analyze. These neighbourhoods all have a very low quantity of reviews associated with them - while the average neighbourhood contains review text amounting to approximately 2.2 million words, none of these neighbourhoods surpassed 300 thousand words, with the average among them being just 80 thousand.

Figure 7 shows in bold the neighbourhoods with the highest stability. These are clustered in the western part of the downtown area, with the Annex neighbourhood showing the highest stability overall (0.9821). Home to Jane Jacobs for the last decades of her life and nearby the University of Toronto, this area was in the past a centre of Toronto's alternative scenes and continues to have one its highest concentrations of artists and young people - even as home prices have risen dramatically in recent years [71, 72, 73]. While it and most of the other bolded neighbourhoods downtown are generally recognized as some of the most dynamic parts of Canada's creative economy, this result indicates that despite 
this - or perhaps because of it - their meaning in the collective imaginary of the city has remained highly stable throughout the period under study.

This stability result overlaps other research using more conventional data sources [74]. Silver's approach for example uses harmonized census data to examine neighbourhood change in Toronto. This technique finds a similar pattern to ours, with the most "creative" neighbourhoods in terms of population young, artistic, creative class workers - remaining highly stable. By contrast, inner suburban areas showed a higher probability of transitioning to alternative neighbourhood types. Combined, these findings suggest that the underlying semantic structure of the downtown core of the city remains mostly stable when compared to its further reaches.

\section{Temporal Analysis: Inter-neighbourhood Change}

We can use the same metrics defined in the previous section to compare the change in different neighbourhoods year on year. This allows us to measure how, for any pair of neighbourhoods, they become more or less semantically similar over time. Figure 9 provides an example of this, showing two neighbourhoods - Pleasant View and North St. James Town - which become distinctly more similar over time.

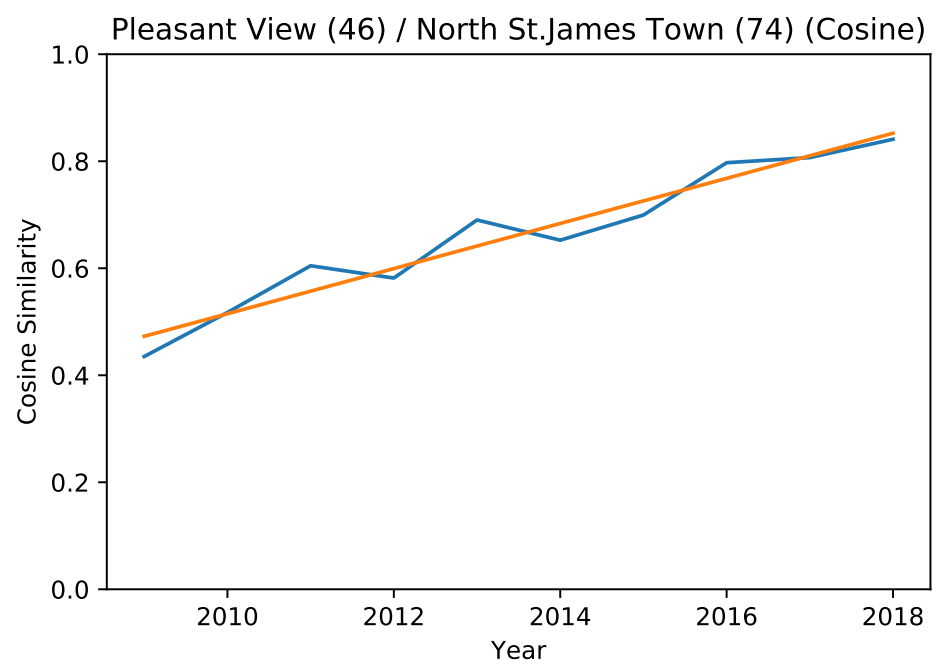

Figure 9: Cosine similarity between embeddings for Pleasant View and North St. James Town over 9 years. Blue line represents original cosine values, orange line represents linear fit.

Unfortunately, when comparing two neighbourhoods in this way, it is difficult to easily ascertain which neighbourhood is responsible for what part of 


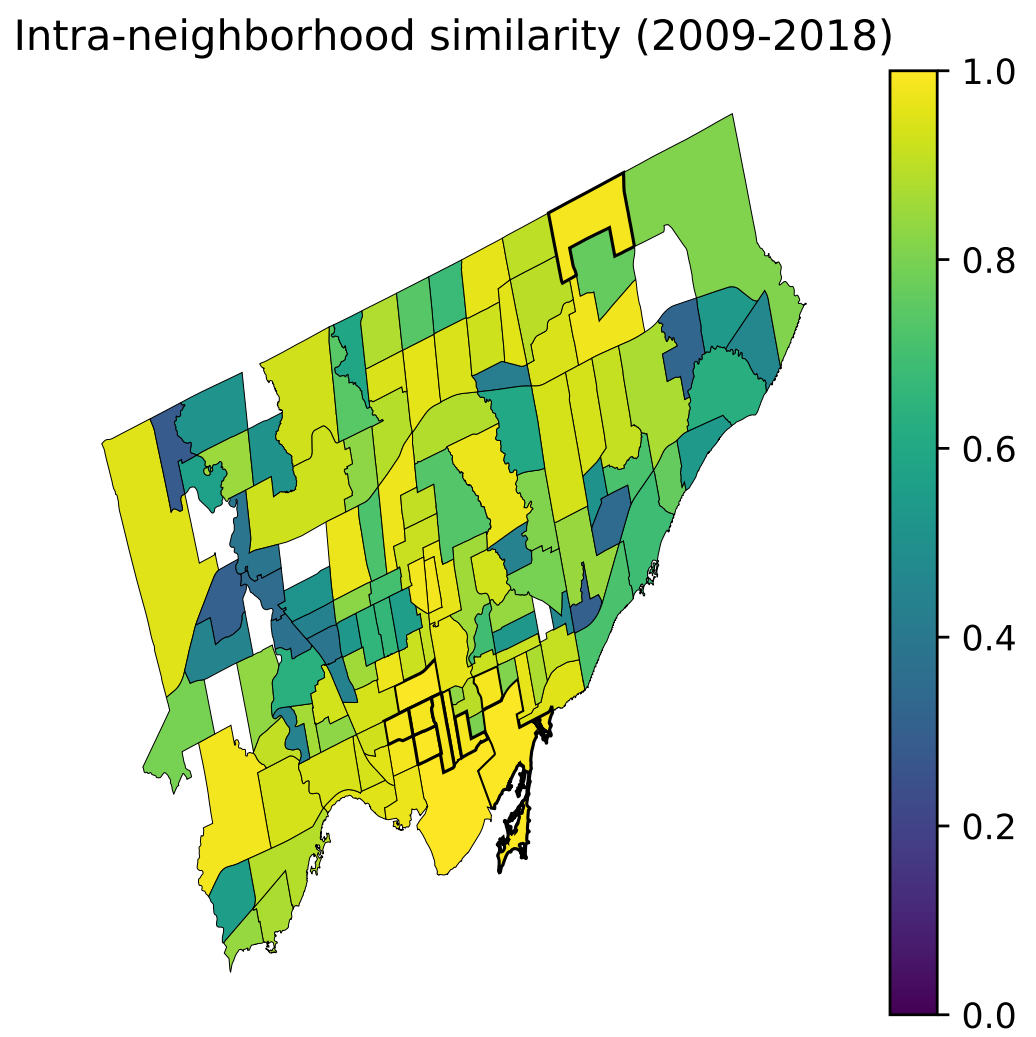

Figure 8: neighbourhood Consistency (2009-2018). A value of 1.0 represents no motion at all through embedding space, while a value of -1 would correspond to total orthogonality to the initial vector (Note that all values here are positive, indicating some minimal similarity with the original vector is always present in this dataset). Bold neighbourhoods are the top 10 most stable. 


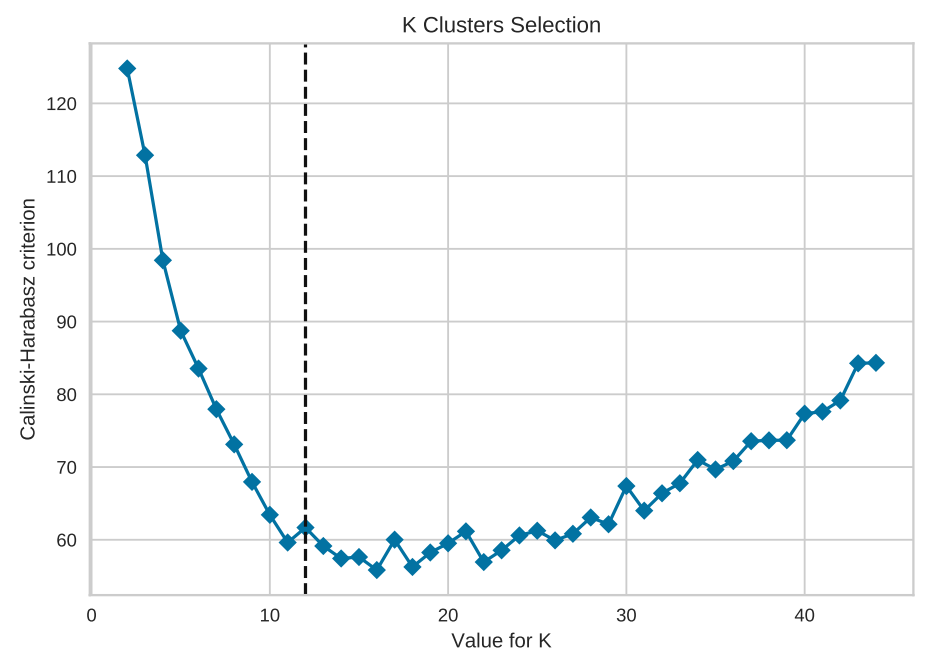

Figure 10: Identifying the optimal clustering $K$ for neighbourhood motion using the CalinskiHarabasz criterion.

the motion. Two neighbourhoods moving slowly together may appear the same as one neighbourhood moving quickly towards another. Additionally, with this metric of comparison, it is difficult to plot more than one pair of neighbourhoods on a single graph.

As we wish to compare all neighbourhoods simultaneously, we need to introduce a "ground truth" to which all neighbourhoods can be compared. For this purpose, we select the most stable neighbourhood from the previous section the Annex. We consider this a strong candidate for the "ground truth" as while this neighbourhood does contain its own information, its extremely low motion through the embedding space allows us to examine the motion of other neighbourhoods relative to this very stable Annex embedding.

\section{Temporal Analysis: Clustering neighbourhood Change}

In order to identify underlying trends in the motion graphs, we utilize KMeans Clustering [75], to group together similar motion. In order to do so, K-Means iteratively assigns each motion graph to a cluster with the most similar average motion, recalculating this average motion and reassigning motion graphs until convergence. In order to select an appropriate number of clusters, $K$, we employ the Calinski-Harabasz criterion [76], which is defined as: 


$$
\frac{S S E_{B}}{S S E_{W}} \times \frac{N-k}{k-1}
$$

Where $N$ is the number of data points (here 139), $k$ the number of clusters, and $S S E_{B}$ and $S S E_{W}$ respectively the between- and within-cluster Sum Squared Error. The Calinski-Harabasz criterion is well established as a suitable metric with which to identify an appropriate value for $\mathrm{K}$ in clustering tasks (e.g. [77, 78, 79]). Figure 10 shows the Calinski-Harabasz score for potential values of $\mathrm{K}$, and we select the value for $\mathrm{K}$ at the knee point, defined as the point of maximum curvature in a line [80]. This gives us a value of $K=12$, which we then use to plot motion graphs separately. These different types of motion are then presented in Figure 11, and the clusters are shown on a map in Figure 12.

Figure 4 shows that neighbourhoods cluster into a few core types of motion, relative to the stable meaning of the Annex. Each line represents a neighbourhood, and points closer to the left side of the graph are more similar to the Annex. Accordingly, Cluster 4 is a set of neighbourhoods that are both relatively stable and represented similarly to the Annex throughout. For these neighbourhoods, the 2009 and 2018 vectors are so similar that representing the 2018 neighbourhood with the vector from 2009, would lose almost no information. Cluster 0 neighbourhoods are also stable but are not as similar to the Annex. Cluster 8 neighbourhoods - a small group on the outskirts of the city - start highly dissimilar to the Annex but have grown steadily more similar to it over time. Major immigrant landing areas, these neighbourhoods correspond to locations in which significant change has been ongoing. Cluster 2 areas also became more similar to the Annex, though they have retained a more distinct representation. Cluster 9 neighbourhoods have become less similar to the Annex in recent years. Cluster 1 and Cluster 7 - geographically near each other - each exhibit momentary "shocks" in which they temporarily and drastically take on very distinct meanings, first in 2011 (in Cluster 1) and second in 2012 (Cluster 7).

Fully articulating and interpreting these different trajectories in relation to their underlying business, review, and user features, as well as other demographic information, goes beyond the scope of this paper. Nevertheless, Figure 7 constitutes a strong affirmative answer to our second research question. Yelp data do provide a powerful window into the temporal evolution of the meaning of urban neighbourhoods. 


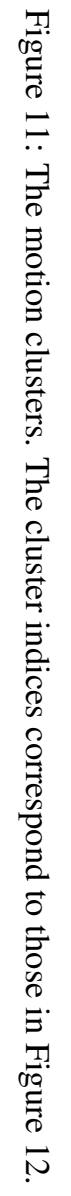

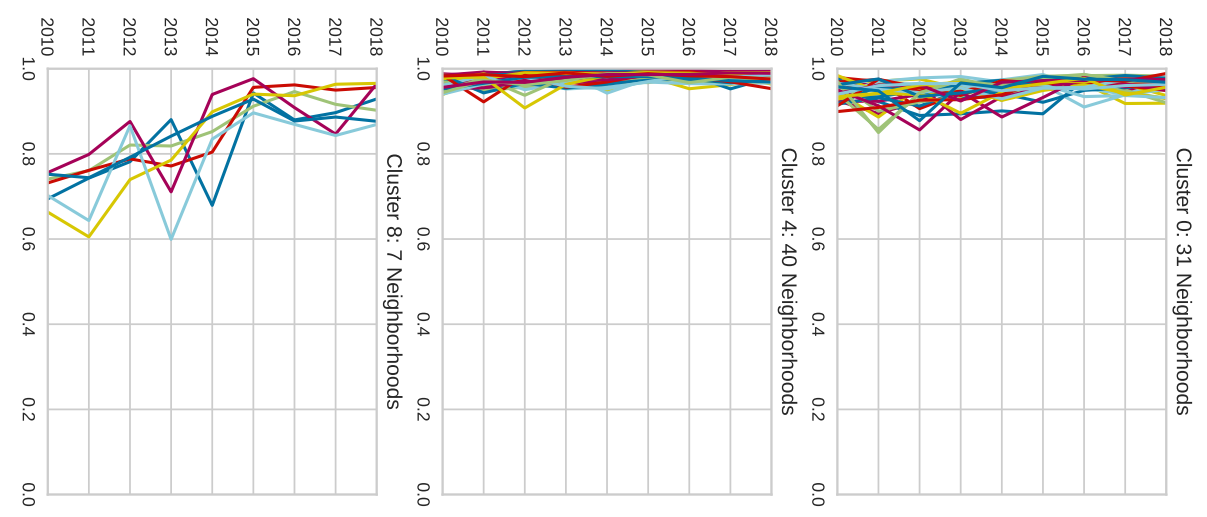

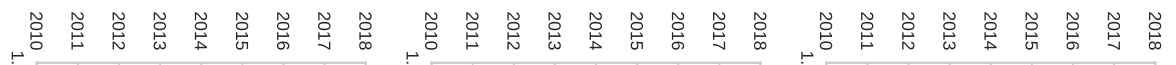
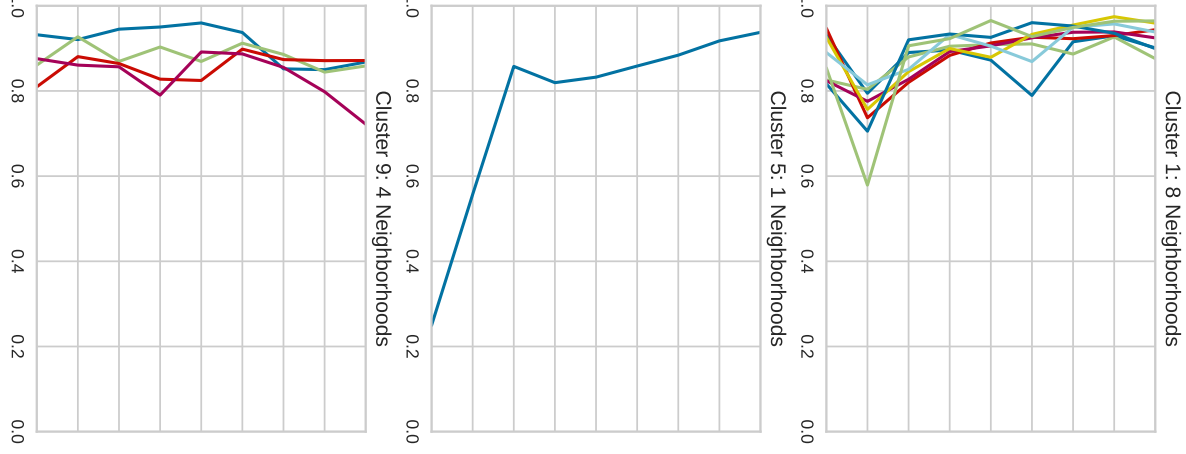

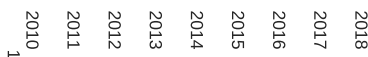
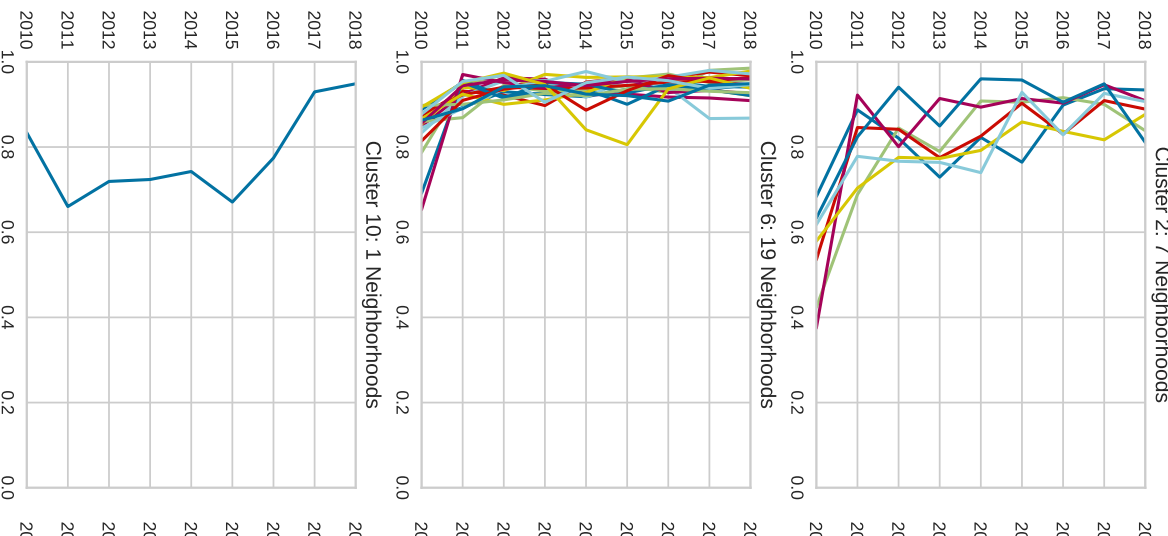

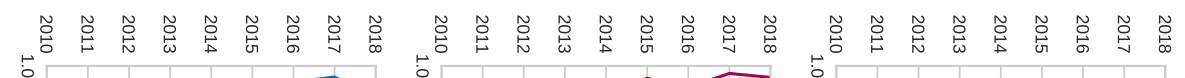

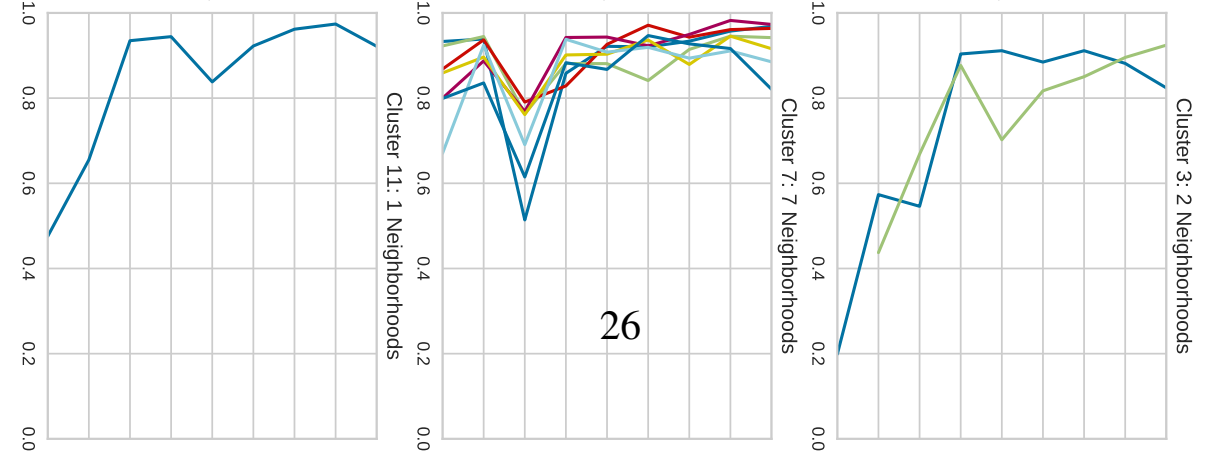




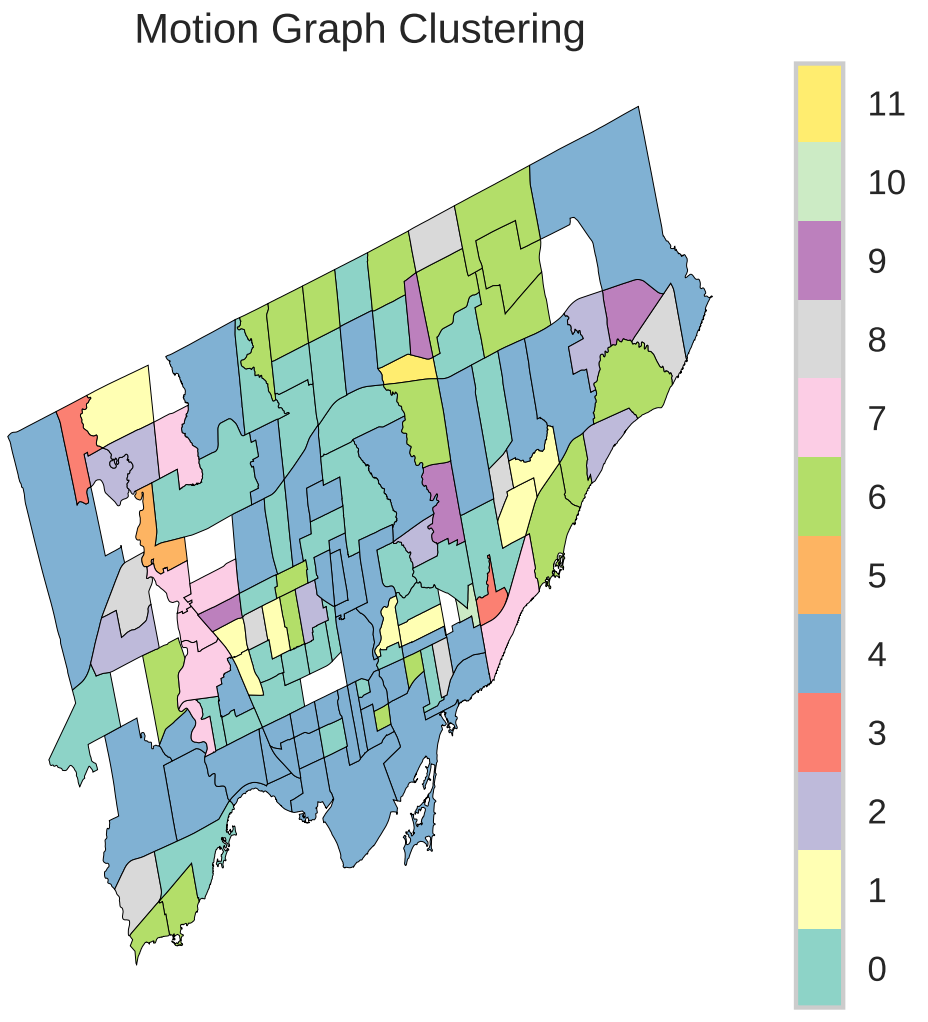

Figure 12: The clusters selected by K-Means clustering on motion graphs. Each color represents a distinct cluster, and the colorings chosen are not related to each other. The cluster indices correspond to those in Figure 11 . 


\section{Discussion}

In this paper, we present a novel methodology for uncovering linguistic structures of cities via social media review texts. In this section, we discuss the implications of this contribution, as well as our experiments.

\section{Real vs Fake neighbourhoods}

The high degree of accuracy that the classifier was able to obtain in the low dimensional space demonstrates the validity of using this review space to examine neighbourhoods. It is important to emphasize that the 'fake' neighbourhoods are indeed real, contiguous sections of the city, meaning that the model was able to distinguish at a high conceptual level the differences between these two sets. Reviews reliably map onto the real neighbourhoods, and the official neighbourhoods exhibit coherent meanings.

Nevertheless, not all neighbourhoods were accurately classified. There are several possible explanations for the incorrectly classified neighbourhoods. For one, due to the random nature of the fake neighbourhood generation, it is entirely possible to accidentally generate a 'fake' neighbourhood, which in reality overlaps significantly with a real neighbourhood. In this situation, it is reasonable for the model to misclassify the fake neighbourhood as real.

In the other direction, regarding real neighbourhoods that are misclassified as fake, such neighbourhoods mainly occur in suburban areas of the city. It is possible that these areas, which often have fewer businesses and reviews when compared to the downtown core of Toronto, are noisier and so appear less 'real' to the model. This is an area for further research.

The broader significance of this analysis emerges when we compare real and fake neighbourhoods in the full-dimensional space, vs that of the embedding, as in Figure 13. This graphic depicts how distinguishing real and fake neighbourhoods in the embedding space is far easier, even upon visual inspection, than it is in the full-dimensional space. This reinforces that the type of analysis we have developed is not possible in the original word frequency space, and that the learned embedding does indeed extract latent information critical to our analyses.

\section{Stable neighbourhoods}

The stable neighbourhood result (Figure 8) demonstrates that especially the west-end downtown centre of Toronto has the most coherent identity compared to the more volatile surrounding areas. The exception to this is Miliken, the only top MSN not in central Toronto, which is located in the far Northeast part of 

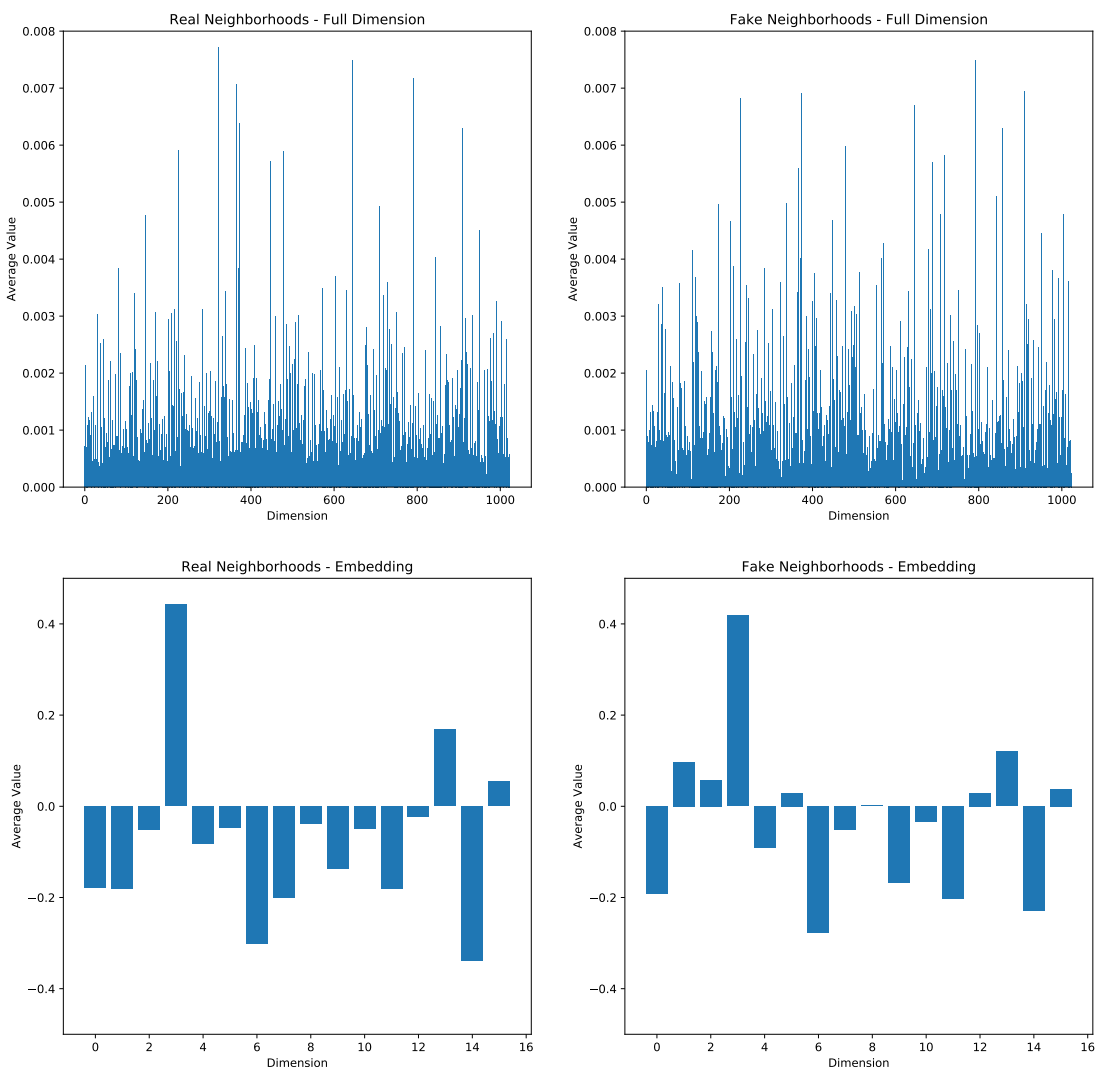

Figure 13: Comparison of the average values for each dimension in the full, 1024-dimensional space, as well as the 16-dimensional embedding space, for real and fake neighbourhoods. The distinction between values in the embedding space is much easier to identify, and these distinctions can be significantly larger, which allows the model to identify fake neighbourhoods far better in the embedding space than it can in the full space. 
the city in bold in Figure 8 . This neighbourhood contains a significant number of business parks, built during the 1990s, which are likely responsible for the neighbourhood's stability in the 2010s.

This ability to identify areas with coherent and stable meaning on the basis of review texts is a central contribution of this paper. Most research uses qualitative and survey methods to identify shared local meaning, often suggesting linkages between neighbourhood identity, solidarity, and local advocacy. Our techniques capture similar information about identity and a platform for pursuing such linkages, but in a way that is scalable, reliable, and more systematically comparable.

An additional benefit of our approach concerns the fact that even the most stable neighbourhoods do have vocabulary change, despite minimal semantic change. This is a key feature of the embedding space: the vocabulary itself is discarded, and meaning is retained. However, as a result of this, it is critical to note that individual words may easily change year-on-year, even in the most stable neighbourhood. The collective meaning of an area transcends changes in specific words, such that a neighbourhood like the Annex is consistently recognized as having a similar meaning within the discursive space of the city, even if specific words used to describe it change. This is an example of how the computational analysis pursued here carries forward key ideas from cultural research, namely about the holistic and relational character of meaning [49, 6].

\section{Motion graph clustering}

The motion graph clustering results, shown in Figures 11 and 12 , clearly show that by reading the city, we can identify a range of different neighbourhood motions, as discussed above.

The confluence of geography and discursive meaning is particularly striking. Cluster 7, for example, contains a group of neighbourhoods tightly compacted together in the west end of Toronto (this is the Weston neighbourhood). This is despite the total lack of proximity information provided to the model. Patterns of meaning are not randomly distributed in space but tend to concentrate nearby and move along similar trajectories. Indeed, these neighbourhoods are all similar in the dissimilarity spike they all experience in 2012. This dissimilarity spike highlights the necessity of grouping motion graphs together. On their own, each motion graph might appear to have some noise causing the graph to change erratically. However, when clustered, it becomes apparent there was some activity in the west of Toronto in 2012, which caused this cluster of neighbourhoods to all rapidly change in the same way. ${ }_{4}^{4}$

\footnotetext{
${ }^{4}$ There are a number of clusters, most notably 5, 10 and 11 , which contain a very small set
} 


\section{Conclusion}

In this paper, we have introduced a methodology for overcoming some of the challenges associated with the empirical analysis of collective representations of urban spaces. These techniques might be considered a form of sociological observatory to complement recent uses of Yelp data for economic monitoring. The economic version uses social media data to identify patterns and trends in business activity before or beyond what official government data permit [7, 8, 9, 45]. Our sociological version uses similar data to identify patterns and trends in collective representations and identities. As a result of this, it expands the range of approaches available to researchers, allowing for more nuanced and effective research of neighbourhood change.

The methods presented here enable a range of novel quantitative analyses. For example, the fake neighbourhood experiment demonstrates that the embeddings capture latent information in the reviews, which are not easily accessible by reading directly (e.g. [81]). Further techniques reveal the relative temporal evolution of neighbourhoods' meaning within the city's discursive space. These techniques open up opportunities for investigating urban processes such as gentrification in a holistic way. For instance, a neighbourhood receiving increasing investments in recent years like Weston [82] — part of Cluster 7 in Figures 11 and 12 - can be identified as becoming more like the Annex, an already gentrified neighbourhood. Findings like these allow us to ascribe tangible, interpretable physical neighbourhood change to these business review embeddings. Such validation shows that the work of the article goes beyond mathematical diversion, and instead is the genesis of a new computational methodology for identifying meaningful neighbourhood change through the lens of denizen-generated textual artifacts.

Developing techniques to uncover the causes and meanings of these and the other changes depicted in Figure 8 is an essential area of further research. Equally important is comparing these discursive trajectories to the sorts of trajectories revealed in the work of Delmelle [83, 84], which is based on demographic trends in, for instance, income, occupation, ethnicity, and age. The interplay between the two - demographic and discursive - raises questions for future research. For instance, we can ask which discursive patterns are most associated

of neighbourhoods. This is a valid result, as the motion graphs for these neighbourhoods do not closely correspond to those of any other neighbourhoods. It is important for our model to be able to correctly identify these neighbourhoods as outliers from general trends so that the trends which $d o$ exist are not ignored in favour of ensuring some minimum cluster size. 
with which demographic patterns, which tend to shift before the other in various times and places, the extent to which one remains stable as the other changes, and more. The techniques developed in this paper put pursuing such questions squarely on the research agenda.

Other opportunities for future research involve further extending the use of modern computational tools. For example, even though the embedding process described here reliably identifies neighbourhoods and their trajectories, due to their highly compact form, their interpretability remains somewhat opaque. Developing tools for unpacking their meaning is a crucial task for future research. Likewise, we can pursue techniques that remove the need to use official neighbourhood boundaries as a baseline. Indeed, other research has suggested that social media data can be used to identify emergent neighbourhood boundaries that arise from out of activity patterns themselves [28, 35]. In order to scale up the techniques developed here to many more cities - another important future step - such methods will be crucial.

Perhaps the most exciting avenue involves moving from description to prediction. Can information about a neighbourhood in Year N, combined with its reviewers and constituent reviews, be used to estimate the direction the neighbourhood can be expected to change in the following year? As a first step toward realizing this goal, novel techniques will be required for identifying what changes in reviews, users, or businesses in the past lead to changes in neighbourhood identity in the future. Building on such techniques, we believe that it may indeed be possible to track motion through the embedding space into the future. Given the embeddings' power to identify phenomena from the past, such as specific types of urban development processes, the applicability of this toolkit to the future may enable researchers to identify trends in neighbourhood change before they even complete. In this way, we can identify not only areas poised to change, but also the type and nature of the change they are likely to undergo. This would constitute not only a sociological observatory and "early warning system" for neighbourhood change, but a genuinely predictive model of urban evolution.

\section{References}

[1] L. R. Ford, Reading the skylines of american cities, Geographical Review 82 (2) (1992) 180-200. URL http://www . jstor .org/stable/215431

[2] H. Wirth-Nesher, Impartial maps: reading and writing cities, Handbook of urban studies (2001) 52-66.

[3] J. M. Leach, R. A. Mulhall, C. D. Rogers, J. R. Bryson, Reading cities: Developing an urban diagnostics approach for identifying integrated urban problems 
with application to the city of birmingham, uk, Cities 86 (2019) 136 - 144. doi:https://doi.org/10.1016/j.cities.2018.09.012. URL http://wWw.sciencedirect.com/science/article/pii/ S0264275118303093

[4] R. Barthes, Semiology and the urban, The city and the sign: An introduction to urban semiotics 8 (1986) 7-98.

[5] K. Lynch, The image of the city, Vol. 11, MIT press, 1960.

[6] G. D. Suttles, The social construction of communities, University of Chicago Press, 1972.

[7] E. L. Glaeser, H. Kim, M. Luca, Nowcasting the local economy: Using yelp data to measure economic activity, Tech. rep., National Bureau of Economic Research (2017).

[8] D. Arribas-Bel, J. Bakens, Use and validation of location-based services in urban research: An example with Dutch restaurants, Urban Studies 56 (5) (2019) 868-884.

[9] L. Dong, C. Ratti, S. Zheng, Predicting neighborhoods' socioeconomic attributes using restaurant data, Proceedings of the National Academy of Sciences 116 (31) (2019) 1544715452.

[10] C. N. Stone, R. P. Stoker, J. Betancur, S. E. Clarke, M. Dantico, M. Horak, K. Mossberger, J. Musso, J. M. Sellers, E. Shiau, et al., Urban neighborhoods in a new era: Revitalization politics in the postindustrial city, University of Chicago Press, 2015.

[11] J. Cranshaw, R. Schwartz, J. Hong, N. Sadeh, The livehoods project: Utilizing social media to understand the dynamics of a city, in: Sixth International AAAI Conference on Weblogs and Social Media, 2012.

[12] G. Filomena, J. A. Verstegen, E. Manley, A computational approach to the image of the city, Cities 89 (2019) 14-25.

[13] M. Horak, A. Moore, Policy Shift without Institutional Change: The Precarious Place of Neighborhood Revitalization in Toronto, in: C. N. Stone, R. P. Stoker (Eds.), Urban Neighborhoods in a New Era: Revitalization Politics in the Postindustrial City, 2015, pp. 182208.

[14] R. J. Sampson, Great American City: Chicago and the Enduring Neighborhood Effect, The University of Chicago Press, Chicago and London, 2012.

[15] N. E. Phillips, B. L. Levy, R. J. Sampson, M. L. Small, R. Q. Wang, The social integration of american cities: Network measures of connectedness based on everyday mobility across neighborhoods, Sociological Methods \& Research (2019) 0049124119852386.

[16] E. Talen, Neighborhood, Oxford University Press, 2018.

[17] J. Hwang, The social construction of a gentrifying neighborhood: Reifying and redefining identity and boundaries in inequality, Urban Affairs Review 52 (1) (2016) 98-128. doi: 10.1177/1078087415570643. URL https://doi.org/10.1177/1078087415570643

[18] C. J. Coulton, J. Korbin, T. Chan, M. Su, Mapping residents' perceptions of neighborhood boundaries: a methodological note, American journal of community psychology 29 (2) (2001) 371-383.

[19] M. Jenks, N. Dempsey, Defining the neighbourhood: Challenges for empirical research, Town Planning Review 78 (2) (2007) 153-177.

[20] J. Portugali, Complexity, cognition and the city, Springer Science \& Business Media, 2011.

[21] J. Jones, The city as text: Reading paris in rayuela, Revista Canadiense de Estudios Hispánicos (1991) 223-234.

[22] L. Pelaschiar, The city as text. the case of belfast: from gothic horror-story to post-modern 
novel.

[23] G. Genette, M. Maclean, Introduction to the paratext, New literary history 22 (2) (1991) 261-272.

[24] R. B. Goh, B. S. Yeoh, Theorizing the Southeast Asian City as Text: Urban Landscapes, Cultural Documents, and Interpretive Experiences, World Scientific, 2003.

[25] J. S. Duncan, The city as text: the politics of landscape interpretation in the Kandyan Kingdom, Cambridge University Press, 2005.

[26] P. A. Longley, M. Adnan, Geo-temporal twitter demographics, International Journal of Geographical Information Science 30 (2) (2016) 369-389.

[27] G. Lansley, P. A. Longley, The geography of twitter topics in london, Computers, Environment and Urban Systems 58 (2016) 85-96.

[28] D. Quercia, L. Capra, J. Crowcroft, The social world of twitter: Topics, geography, and emotions, in: Sixth International AAAI Conference on Weblogs and Social Media, 2012.

[29] D. Fried, M. Surdeanu, S. Kobourov, M. Hingle, D. Bell, Analyzing the language of food on social media, in: 2014 IEEE International Conference on Big Data (Big Data), IEEE, 2014, pp. 778-783.

[30] M. Arnaboldi, M. Brambilla, B. Cassottana, P. Ciuccarelli, S. Vantini, Urbanscope: A lens to observe language mix in cities, American Behavioral Scientist 61 (7) (2017) 774-793.

[31] V. Vargas-Calderón, J. E. Camargo, Characterization of citizens using word2vec and latent topic analysis in a large set of tweets, Cities 92 (2019) 187-196.

[32] K. Joseph, C. H. Tan, K. M. Carley, Beyond local, categories and friends: clustering foursquare users with latent topics, in: Proceedings of the 2012 ACM conference on ubiquitous computing, ACM, 2012, pp. 919-926.

[33] T. H. Silva, P. O. V. de Melo, J. M. Almeida, M. Musolesi, A. A. Loureiro, You are what you eat (and drink): Identifying cultural boundaries by analyzing food and drink habits in foursquare, in: Eighth International AAAI Conference on Weblogs and Social Media, 2014.

[34] C. Wagner, P. Singer, M. Strohmaier, The nature and evolution of online food preferences, EPJ Data Science 3 (1) (2014) 38.

[35] D. Arribas-Bel, K. Kourtit, P. Nijkamp, The sociocultural sources of urban buzz, Environment and Planning C: Government and Policy 34 (1) (2016) 188-204.

[36] A. Noulas, S. Scellato, C. Mascolo, M. Pontil, Exploiting semantic annotations for clustering geographic areas and users in location-based social networks, in: Fifth International AAAI Conference on Weblogs and Social Media, 2011.

[37] D. Karamshuk, A. Noulas, S. Scellato, V. Nicosia, C. Mascolo, Geo-spotting: mining online location-based services for optimal retail store placement, in: Proceedings of the 19th ACM SIGKDD international conference on Knowledge discovery and data mining, ACM, 2013, pp. 793-801.

[38] X. Long, L. Jin, J. Joshi, Exploring trajectory-driven local geographic topics in foursquare, in: Proceedings of the 2012 ACM conference on ubiquitous computing, ACM, 2012, pp. 927-934.

[39] F. Zhang, L. Wu, D. Zhu, Y. Liu, Social sensing from street-level imagery: A case study in learning spatio-temporal urban mobility patterns, ISPRS Journal of Photogrammetry and Remote Sensing 153 (2019) 48-58.

[40] N. Naik, J. Philipoom, R. Raskar, C. Hidalgo, Streetscore-predicting the perceived safety of one million streetscapes, in: Proceedings of the IEEE Conference on Computer Vision 
and Pattern Recognition Workshops, 2014, pp. 779-785.

[41] D. Jurafsky, V. Chahuneau, B. R. Routledge, N. A. Smith, Narrative framing of consumer sentiment in online restaurant reviews, First Monday 19 (4).

[42] K. Reschke, A. Vogel, D. Jurafsky, Generating recommendation dialogs by extracting information from user reviews, in: Proceedings of the 51st Annual Meeting of the Association for Computational Linguistics (Volume 2: Short Papers), 2013, pp. 499-504.

[43] B. Kovács, G. R. Carroll, D. W. Lehman, Authenticity and consumer value ratings: Empirical tests from the restaurant domain, Organization Science 25 (2) (2014) 458-478.

[44] A. Goldberg, M. T. Hannan, B. Kovács, What does it mean to span cultural boundaries? variety and atypicality in cultural consumption, American Sociological Review 81 (2) (2016) $215-241$.

[45] E. L. Glaeser, H. Kim, M. Luca, Nowcasting gentrification: using yelp data to quantify neighborhood change, in: AEA Papers and Proceedings, Vol. 108, 2018, pp. 77-82.

[46] S. Zukin, S. Lindeman, L. Hurson, The omnivores neighborhood? online restaurant reviews, race, and gentrification, Journal of Consumer Culture 17 (3) (2017) 459-479.

[47] C. D. Manning, P. Raghavan, H. Schütze, Introduction to Information Retrieval, Cambridge University Press, New York, NY, USA, 2008.

[48] D. M. Blei, A. Y. Ng, M. I. Jordan, Latent dirichlet allocation, Journal of machine Learning research 3 (Jan) (2003) 993-1022.

[49] P. DiMaggio, M. Nag, D. Blei, Exploiting affinities between topic modeling and the sociological perspective on culture: Application to newspaper coverage of us government arts funding, Poetics 41 (6) (2013) 570-606.

[50] A. Edelmann, T. Wolff, D. Montagne, C. A. Bail, Computational Social Science and Sociology, Annual Review of Sociology 46 (2020) 1-24. doi:10.1146/ annurev-soc-121919-054621.

[51] F. Kling, A. Pozdnoukhov, When a city tells a story: urban topic analysis, in: Proceedings of the 20th international conference on advances in geographic information systems, ACM, 2012, pp. 482-485.

[52] J. Pennington, R. Socher, C. Manning, Glove: Global vectors for word representation, in: Proceedings of the 2014 conference on empirical methods in natural language processing (EMNLP), 2014, pp. 1532-1543.

[53] T. Mikolov, K. Chen, G. Corrado, J. Dean, Efficient estimation of word representations in vector space, arXiv preprint arXiv:1301.3781.

[54] Q. Le, T. Mikolov, Distributed representations of sentences and documents, in: Proceedings of the 31st International Conference on International Conference on Machine Learning Volume 32, ICML14, JMLR.org, 2014, p. II1188II1196.

[55] D. Bollegala, C. Bao, Learning word meta-embeddings by autoencoding, in: Proceedings of the 27th International Conference on Computational Linguistics, 2018, pp. 1650-1661.

[56] S. C. AP, S. Lauly, H. Larochelle, M. Khapra, B. Ravindran, V. C. Raykar, A. Saha, An autoencoder approach to learning bilingual word representations, in: Advances in Neural Information Processing Systems, 2014, pp. 1853-1861.

[57] J. Li, M.-T. Luong, D. Jurafsky, A hierarchical neural autoencoder for paragraphs and documents, arXiv preprint arXiv:1506.01057.

[58] B. L. Ranard, R. M. Werner, T. Antanavicius, H. A. Schwartz, R. J. Smith, Z. F. Meisel, D. A. Asch, L. H. Ungar, R. M. Merchant, Yelp reviews of hospital care can supplement and inform traditional surveys of the patient experience of care, Health Affairs 35 (4) (2016) 
697-705.

[59] Y. Yuan, Y. Lu, T. E. Chow, C. Ye, A. Alyaqout, Y. Liu, The missing parts from social media-enabled smart cities: Who, where, when, and what?, Annals of the American Association of Geographers 110 (2) (2020) 462-475.

[60] J. Fogel, S. Zachariah, Intentions to use the yelp review website and purchase behavior after reading reviews, Journal of theoretical and applied electronic commerce research 12 (1) (2017) 53-67.

[61] B. D. Carpenter, S. Buday, Computer use among older adults in a naturally occurring retirement community, Computers in Human Behavior 23 (6) (2007) 3012-3024.

[62] Quantcast, Audience insights (Apr 2020).

URL https://www.quantcast.com/measure/profile/site/p-M4yfUTCPeS3vn/ yelp.co.uk? countryCode=GB\&tab=demographics

[63] J. Johnson, Gb: online audience of yelp.co.uk by age group gender 2020 (Feb 2020). URL https://www.statista.com/statistics/491925/ distribution-of-the-online-audience-of-yelpcouk-in-gb-by-age-group-and-gender/

[64] P. Ruch, R. Baud, A. Geissbühler, Evaluating and reducing the effect of data corruption when applying bag of words approaches to medical records, International Journal of Medical Informatics 67 (1-3) (2002) 75-83.

[65] B. C. How, K. Narayanan, An empirical study of feature selection for text categorization based on term weightage, in: IEEE/WIC/ACM International Conference on Web Intelligence (WI'04), IEEE, 2004, pp. 599-602.

[66] C. A. Martins, M. C. Monard, E. T. Matsubara, Reducing the dimensionality of bag-ofwords text representation used by learning algorithms, in: Proc of 3rd IASTED International Conference on Artificial Intelligence and Applications, 2003, pp. 228-233.

[67] S. Deerwester, S. T. Dumais, G. W. Furnas, T. K. Landauer, R. Harshman, Indexing by latent semantic analysis, Journal of the American society for information science 41 (6) (1990) 391-407.

[68] M. Honnibal, I. Montani, spaCy 2: Natural language understanding with Bloom embeddings, convolutional neural networks and incremental parsing, to appear (2017).

[69] S. Yu, C. Xu, H. Liu, Zipf's law in 50 languages: its structural pattern, linguistic interpretation, and cognitive motivation, CoRR abs/1807.01855. arXiv: 1807.01855 URL http://arxiv.org/abs/1807.01855

[70] F. Pedregosa, G. Varoquaux, A. Gramfort, V. Michel, B. Thirion, O. Grisel, M. Blondel, P. Prettenhofer, R. Weiss, V. Dubourg, J. Vanderplas, A. Passos, D. Cournapeau, M. Brucher, M. Perrot, E. Duchesnay, Scikit-learn: Machine learning in Python, Journal of Machine Learning Research 12 (2011) 2825-2830.

[71] M. Patterson, D. Silver, The place of art: Local area characteristics and arts growth in canada, 2001-2011, Poetics 51 (2015) 69-87.

[72] M. Bowman, Artists in the canadian urban fabric (Jan 2013). URL http://spacing.ca/toronto/2010/03/17/artists-in-the-canadian-urban-fabric/

[73] D. Churchill, American expatriates and the building of alternative social space in toronto, 1965-1977, Urban History Review/Revue d'histoire urbaine 39 (1) (2010) 31-44.

[74] D. Silver, The city is constantly making new versions of itself (Jul 2018). URL https://urbangenome.utoronto.ca/node/36

[75] J. MacQueen, et al., Some methods for classification and analysis of multivariate observations, in: Proceedings of the fifth Berkeley symposium on mathematical statistics and 
probability, Vol. 1, Oakland, CA, USA, 1967, pp. 281-297.

[76] T. Caliński, J. Harabasz, A dendrite method for cluster analysis, Communications in Statistics-theory and Methods 3 (1) (1974) 1-27.

[77] S. Nanda, B. Mahanty, M. Tiwari, Clustering indian stock market data for portfolio management, Expert Systems with Applications 37 (12) (2010) 8793-8798.

[78] N. Czink, P. Cera, J. Salo, E. Bonek, J.-P. Nuutinen, J. Ylitalo, A framework for automatic clustering of parametric mimo channel data including path powers, in: IEEE Vehicular Technology Conference, IEEE, 2006, pp. 1-5.

[79] S. Łukasik, P. A. Kowalski, M. Charytanowicz, P. Kulczycki, Clustering using flower pollination algorithm and calinski-harabasz index, in: 2016 IEEE Congress on Evolutionary Computation (CEC), IEEE, 2016, pp. 2724-2728.

[80] V. Satopaa, J. Albrecht, D. Irwin, B. Raghavan, Finding a" kneedle" in a haystack: Detecting knee points in system behavior, in: 201131 st international conference on distributed computing systems workshops, IEEE, 2011, pp. 166-171.

[81] S. Kay, Opinion: Yelp reviewers' authenticity fetish is white supremacy in action (Jan 2019).

URL

https://ny.eater.com/2019/1/18/18183973/ authenticity-yelp-reviews-white-supremacy-trap

[82] L. Rainford, Toronto's weston area revitalized by housing and transit developments (Oct 2018).

URL https://www .toronto.com/news-story/8946043-toronto-s-weston-area-revitalized-by-hous

[83] E. C. Delmelle, Differentiating pathways of neighborhood change in 50 us metropolitan areas, Environment and planning A 49 (10) (2017) 2402-2424.

[84] E. C. Delmelle, Mapping the DNA of urban neighborhoods: Clustering longitudinal sequences of neighborhood socioeconomic change, Annals of the American Association of Geographers 106 (1) (2016) 36-56. doi:10.1080/00045608.2015.1096188

\section{Appendix A. Yelp Methodology}

While Yelp does make available its own 'academic dataset' for research, this dataset contains only a subset of the businesses available in any given city. For this reason, we decided that it was necessary to obtain our own, more complete version of the Yelp dataset for one city. We chose Toronto in part due to our residence there, but also due to its substantial public dataset availability. The city of Toronto makes available an official set of neighbourhood shapefiles, defining the 140 neighbourhoods considered to exist by the city government (Figure 1). The scraping method for obtaining this data comprises a two-stage process. The first stage employs the official Yelp API to discover all businesses in Toronto, while the second stage uses a scraping script to obtain detailed data about these businesses.

Yelp exposes an official API for developers, which only provides basic information about businesses on the site. Critically, this API only provides the first twenty reviews for any business, selected using a proprietary Yelp relevancy al- 
gorithm. The information provided by this API is sufficient to obtain a list of all businesses in Toronto. However, it is not possible to simply pass an empty search to Yelp to obtain a full list of businesses in a defined region. A more sophisticated approach is required for two reasons: (a) Yelp's API requires some text in the search field, and (b) Yelp only returns the first one hundred businesses that match any query. In order to work within these constraints, the following recursive method was created. For each category officially defined by Yelp (e.g. 'Chinese Restaurant', 'Rock Climbing'), search only the name of the category using the API. Then, if the number of businesses returned is greater than one hundred, sub-divide the boundaries into one hundred grid-based regions. Call the function on each of these regions and append the output to the total list of businesses. Otherwise, return the list of businesses returned by the API for the query. Using this method, we obtained the Yelp listing for every business in Toronto. The number of businesses obtained, approximately 24000 , was validated against results from [7], in which they found that across the US, Yelp had $18.7 \%$ of the listings that the official business patterns dataset did. Our Toronto dataset comprises a similar proportion of all businesses officially registered in Toronto (which includes many non-consumer facing businesses that would not be expected to have Yelp pages). 